\title{
Exploring Early and Late Cosmology with Next Generation Surveys
}

\author{
Guilherme Brando*1,2, Eric V. Linder ${ }^{3,4}$ \\ ${ }^{1}$ PPGCosmo, CCE - Universidade Federal do Espírito Santo, 29075-910, Vitória, ES, Brazil \\ ${ }^{2}$ Institute of Cosmology $\&$ Gravitation, University of Portsmouth, \\ Dennis Sciama Building, Burnaby Road, Portsmouth, PO1 3FX, United Kingdom \\ ${ }^{3}$ Berkeley Center for Cosmological Physics $\&$ Berkeley Lab, \\ University of California, Berkeley, CA 94720, USA \\ ${ }^{4}$ Energetic Cosmos Laboratory, Nazarbayev University, Nur-Sultan, Kazakhstan 010000 *
}

(Dated: May 12, 2020)

Perturbations from inflation evolve into large scale structure of the late universe, and encode abundant cosmic structure formation physics. We allow freedom in the primordial power spectrum, rather than assuming a power law scale dependence, to study its impact on cosmological parameter determination. Combining various generations of cosmic microwave background (CMB) data and galaxy redshift survey data, we investigate the constraints on reconstruction of the primordial curvature perturbation power spectrum and the late time cosmology, especially the sum of neutrino masses. We quantify how each successive generation, in CMB and galaxy surveys, provides significant improvements, often by factors of several. By using CMB polarization information over a broad range of angular scales, and galaxy redshift data in many bins of redshift, one can allow inflationary freedom and still constrain parameters comparably to assuming power law dependence. The primordial power spectrum can be reconstructed at the subpercent level in a dozen wavenumber bins, while simultaneously fitting the sum of neutrino masses to $14 \mathrm{meV}$.

* gbrando@cosmo-ufes.org 


\section{INTRODUCTION}

The vast array of large scale structure in the present universe formed from primordial seeds, possibly laid down as quantum fluctuations during early universe inflation. Cosmic structure is a convolution of the primordial perturbations and the evolution throughout cosmic history (also known as transfer functions). Thus it carries important information on both the early and late universe.

Generally one assumes the form of the primordial perturbations is known, in terms of a power law (or power law plus running) scale dependence, and then measures structure through the cosmic microwave background (CMB) and galaxy surveys. When measuring structure at different cosmic times, one can extract the time dependence of its growth. This is influenced by the cosmic expansion history (and hence dark energy), the matter density (including dark matter), and neutrino mass (which also gives an additional scale dependence).

These are all fundamental quantities of great import. However, one might wonder whether the assumptions about the primordial, i.e. initial, perturbation power spectrum could influence the results. This has been explored in various papers (e.g. [1-19]). Another research area seeks to reconstruct the primordial power spectrum from data (see, e.g., [20-23] and many others). Here we investigate both - allowing inflationary freedom and examining its effect on late time cosmology, while seeing how late time data aids in reconstructing the primordial power spectrum. In particular, CMB data probes the density power spectrum on many scales, while galaxy redshift data can probe it at many times; together they can deconvolve the evolution from the initial conditions.

We emphasize taking a function-free form (which we will refer to as free form) primordial curvature perturbation power spectrum, to allow for features of various sorts (including a power law) from inflation physics. While there are models that predict steps, bends, or oscillations linear or logarithmic in scale (see, e.g., [13, 15, 18, 19, 24-27] and references therein), we work with a free form that would allow the data itself to reconstruct its preferred behavior. While no finite number of free variables gives complete freedom, this does give far more freedom than fixing to power law, and previous literature (e.g. [1]) has established that 20 bins give sufficient freedom for cases of most interest. Of course one would also compare the data to particular compelling models, but without clear guidance the phenomenological approach we take here can provide useful first tests.

Our goal is to consider how the next generation of CMB experiments and galaxy surveys will improve the simultaneous fitting of early and late time cosmology. We look at CMB experiments (present and future) alone, and in combination with next generations of galaxy redshift surveys, with particular focus on reconstructing the primordial power spectrum and the late time constraint on the sum of neutrino masses.

In Sec. II we lay out the method for implementing inflationary freedom, treating the galaxy power spectrum, and analyzing the constraints on inflation, cosmology, and astrophysical parameters. Section III summarizes the surveys we will consider, of various generations, and the types and range of data used. In Sec. IV we discuss the results of various combinations of data, by probe and generation, and summarize the prospects for reconstruction of the inflationary power spectrum and constraint on the sum of neutrino masses. We conclude in Sec. V.

\section{INFLATIONARY FREEDOM AND COSMOLOGY}

The growth of large scale structure can be viewed as a convolution of the initial conditions, in terms of the primordial curvature perturbation power spectrum $\Delta_{\mathcal{R}}^{2}$, with a scale and time dependent transfer function $T(k, z)$ describing the evolution:

$$
P_{k}^{\delta}(z)=\Delta_{\mathcal{R}}^{2}(k) * T(k, z)
$$

where $\mathcal{R}$ is the curvature perturbation and $\delta$ is the fractional matter density perturbation, with $k$ the Fourier mode and $z$ the redshift. For the remainder of the article we will write $P_{k}^{\delta}$ simply as $P_{k}$.

From this form we can immediately see that data $P_{k}(z)$ at several redshifts allows the possibility of separating the primordial contribution from the evolution, and measuring both. Using the CMB at the redshift of last scattering $z_{\text {lss }} \approx 1090$ and galaxy surveys in several redshift slices $z \lesssim 5$ in principle permits simultaneous constraints on the early and late universe. In particular, future galaxy surveys that extend to $z \gtrsim 2$ and have multiple redshift bins should have large numbers of linear modes - even larger than the number of modes mapped by the CMB - hence with greater statistical leverage and easier to interpret, and a range of redshifts to separate out the several cosmological influences.

The scale dependence in the transfer function generally comes from when perturbation modes enter the horizon (i.e. radiation vs matter dominated epoch), which is well accounted for in Boltzmann codes, from nonlinear mode coupling (which we will avoid by restricting to the linear density perturbation regime), and from scale dependent cosmology such as the influence of neutrino mass (which we include). The time dependence involves the cosmic background 
expansion, and hence the matter density and dark energy properties. We will work within the flat $\Lambda$ CDM model to focus on the matter density, neutrino mass, and mass fluctuation amplitude $\sigma_{8}$.

Conventionally one assumes a particular form for $\Delta_{\mathcal{R}}^{2}(k)$, most usually a power law in $k$, sometimes with another parameter describing the running of the power law index. That is,

$$
\Delta_{\mathcal{R}, 0}^{2}(k)=\Delta_{\mathcal{R}, 0}^{2}\left(k_{\text {piv }}\right)\left(\frac{k}{k_{\text {piv }}}\right)^{n_{s}-1+\left(\alpha_{s} / 2\right) \ln \left(k / k_{\text {piv }}\right)}
$$

where $k_{\text {piv }}$ is a pivot wavenumber, $n_{s}$ the power law index, and $\alpha_{s}$ the running. While the simplest theories of inflation predict such a form, interest has increased in forms that could include oscillations or other features. Therefore we want to allow great inflationary freedom. Since there is particular interest recently in oscillations, we avoid adopting any form that might give spurious oscillations for ringing, such as splines, principal components, or wavelets.

For the primordial power spectrum (PPS) we use 20 bins in $k$, giving a highly model independent form capable of reconstructing oscillations or features. Thus the PPS becomes

$$
\Delta_{\mathcal{R}}^{2}(k)=\Delta_{\mathcal{R}, 0}^{2}(k)\left[1+\sum h_{i} B_{i}(k)\right]
$$

where $B_{i}$ is the top hat function ( 1 inside the bin centered at $k_{i}, 0$ outside), and $h_{i}$ is the free parameter giving the height of the deviation from the fiducial power law. That is, we allow the power law PPS to be multiplied in each bin by a factor $1+h_{i}$, and sample $h_{i}$ with a uniform prior in $[-0.8,0.8]$. We set $\Delta_{\mathcal{R}, 0}^{2}$ by adopting the fixed Planck 2018 [28] values of $A_{s}=2.1 \times 10^{-9}$ (which determines $\Delta_{\mathcal{R}, 0}^{2}\left(k_{\text {piv }}\right)$ according to our conventions), $n_{s}=0.966$, and $\alpha_{s}=0$ for $k_{\text {piv }}=0.05 \mathrm{Mpc}^{-1}$. The bins are spaced logarithmically in $k$, from $10^{-3}-10^{-0.5} \mathrm{Mpc}^{-1}, 2.5$ decades with 8 bins per decade so 20 bins total. To recap: the $h_{i}$ give the amplitude in each bin, and if they "work together" they can choose an overall slope. The data determines this, the form Eq. (3) does not impose it.

The matter density power spectrum $P_{m}$ is determined from the primordial curvature power spectrum through solution of the evolution equations within an appropriately modified version of the CLASS Boltzmann code [29, 30] (specifically, we generalize the initial conditions by introducing our binned PPS version in the code via the external_Pk mode feature and add a galaxy clustering likelihood module for the future data we use). We convert this to the galaxy power spectrum by including galaxy bias and redshift space distortions, as well as a nuisance contribution from nonlinear density effects and deviations from linear galaxy bias. We write the galaxy power spectrum $P_{g}$ as

$$
P_{g}(k, \mu, z)=\left[b(z)+f(z) \mu^{2}\right]^{2} P_{m}(k, z)+P_{0}(z)
$$

where $b(z)$ is the linear bias factor, $f(z)$ is the matter growth rate, $\mu$ is the cosine of the angle of the Fourier mode $\vec{k}$ with respect to the line of sight, and $P_{0}$ is a nuisance function allowing for power coming from misestimation of bias, nonlinearities, and shot noise. This form follows that used in analyses of BOSS galaxy redshift survey data [1, 31].

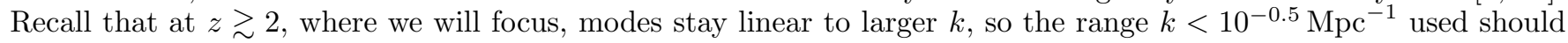
be reasonably described by linear theory.

The growth rate $f(z)$ will be determined by the background cosmological parameters (since we are here only using data at $z \gtrsim 2$ we use flat $\Lambda$ CDM), and we include parameters $b_{j}$ and $S_{j}$ for each redshift bin of the survey, where $S_{j} \equiv n_{j} P_{0}\left(z_{j}\right)$. For $b_{j}$ we take uniform priors in $\left[b_{j, \text { fid }}-1.5, b_{j, \text { fid }}+1.5\right]$, where the fiducial value depends on the survey (see the next section). For $S_{j}$ we take uniform priors in $[-0.5,0.5]$ for the additional, nuisance power. $\left(\right.$ Note $S_{j}=n_{j} P_{0}$ is from the unaccounted-for extra nuisance power, and is not the $n P_{g}$ of the survey.) The cosmological parameters are the dimensionless physical baryon density $\Omega_{b} h^{2}$, dimensionless physical cold dark matter density $\Omega_{c d m} h^{2}$, Hubble constant $H_{0}$ (written in units of $\mathrm{km} \mathrm{s}^{-1} \mathrm{Mpc}^{-1}$ ), sum of neutrino masses $\sum m_{\nu}$, and optical depth $\tau$. Recall that $A_{s}$ and $n_{s}$ are replaced as free parameters by the twenty $h_{i}$. Derived parameters may include the total matter density in units of the critical density, $\Omega_{m}$, and the mass fluctuation amplitude $\sigma_{8}$.

\section{NEXT GENERATION SURVEYS}

One main focus is to explore how next generation surveys, of both the CMB and large scale structure clustering, will improve our knowledge of the primordial power spectrum, without assuming a functional form for it, i.e. allowing inflationary freedom. We consider two CMB surveys: the current Planck 2018 data and a future CMB-S4 experiment $[32,33]$. For galaxy redshift surveys, we consider two variations of the proposed next generation MegaMapper survey, called the Ideal and Fiducial versions [34, 35].

The Planck data is used through the Planck 2018 likelihood code available at MontePython [36, 37]. We use the Planck18 baseline likelihood: Planck TTTEEE, Planck low-T, low-E, and lensing. 
For the CMB-S4 experiment we create a mock data set assuming Planck 2018 bestfit $\Lambda$ CDM parameter values $\left(\Omega_{b} h^{2}=0.02238, \Omega_{c d m} h^{2}=0.1201, H_{0}=67.32, \tau_{\text {reio }}=0.0543, n_{s}=0.966, A_{s}=2.1 \times 10^{-9}, \sum m_{\nu}=0.06 \mathrm{eV}\right)$, and we use the mock likelihood class for this future survey introduced in [38] (note that CMB-S4 experiment characteristics may have changed since then). Note that for CMB-S4 we do not add multipoles $\ell<30$ outside its range (i.e. do not also use Planck18 data), which, together with using a realization of the $\Lambda$ CDM model rather than measured data, can shift the best fit parameters relative to Planck18 data fits.

MegaMapper mock data likelihood was created through a galaxy clustering likelihood module added to MontePython through a class module montepython/likelihoods_class.py, using the survey characteristics described in [35] including galaxy bias $b_{j, \text { fid }}$ and galaxy number density $n_{j}$ entering the shot noise added to the data. Both Ideal and Fiducial cases span the range $z=2-5$, though with a higher galaxy number density and finer redshift binning in the Ideal case (seven and four bins respectively, each bin with parameters for galaxy bias $b_{j}$ and modeling $S_{j}$ ).

Thus we have 20 inflation parameters, 5 cosmology parameters (i.e. the PPS amplitude $A_{s}$ and power law index $n_{s}$ are replaced by the 20 inflation parameters), and 8 or 14 astrophysical nuisance parameters, in addition to CMB nuisance parameters.

Our scientific focus here is mainly on two aspects of the physics: the primordial power spectrum and the sum of the neutrino masses, though we will discuss the other parameter constraints, especially any unusual ones. We will analyze cases of data combinations in both a "probe" - CMB only or CMB plus galaxy redshift survey - and a "generational" sense - current data, moderately future data (including MegaMapper Fiducial data), and further future data (including CMB-S4 or MegaMapper Ideal).

\section{RESULTS}

\section{A. CMB only}

We begin with just the CMB data, and the current data from the Planck 2018 data set, which includes the lowest multipoles, important for constraining the small $k$ modes of the primordial power spectrum. The PPS reconstruction is shown in Fig. 1. In general this agrees well with a power law, with the slope $n_{s}$ consistent with the usual power law Planck results. The significant, well-known deviation is in the third free bin of amplitude, $h_{3}$ at $k \in[1.78,2.37] \times$ $10^{-3} \mathrm{Mpc}^{-1}$. This is lower than the power law expectation at more than $95 \%$ confidence level (CL), and corresponds to the dip in the temperature power spectrum data around $\ell \approx 20$. Since there is freedom in the other $k$ bins, they rise slightly in amplitude to compensate, but still follow a very similar power law dependence.

The 20 PPS bin amplitude parameters $h_{i}$ are given in Table I and plotted in Fig. 2. (All plots of posteriors in this work were generated using the Python package GetDist [39].) Except for $h_{3}$ they are all consistent with zero (i.e. the standard power law PPS) at 95\% confidence level. The $68 \%$ CL constraints range from uncertainties of $\sim 1.5 \%$ in $1+h_{i}$ (see Eq. 3) at the tightest to $\sim 50 \%$ at the smallest $k$ (where cosmic variance is large) and largest $k$ (where Planck measurement precision begins to weaken). 


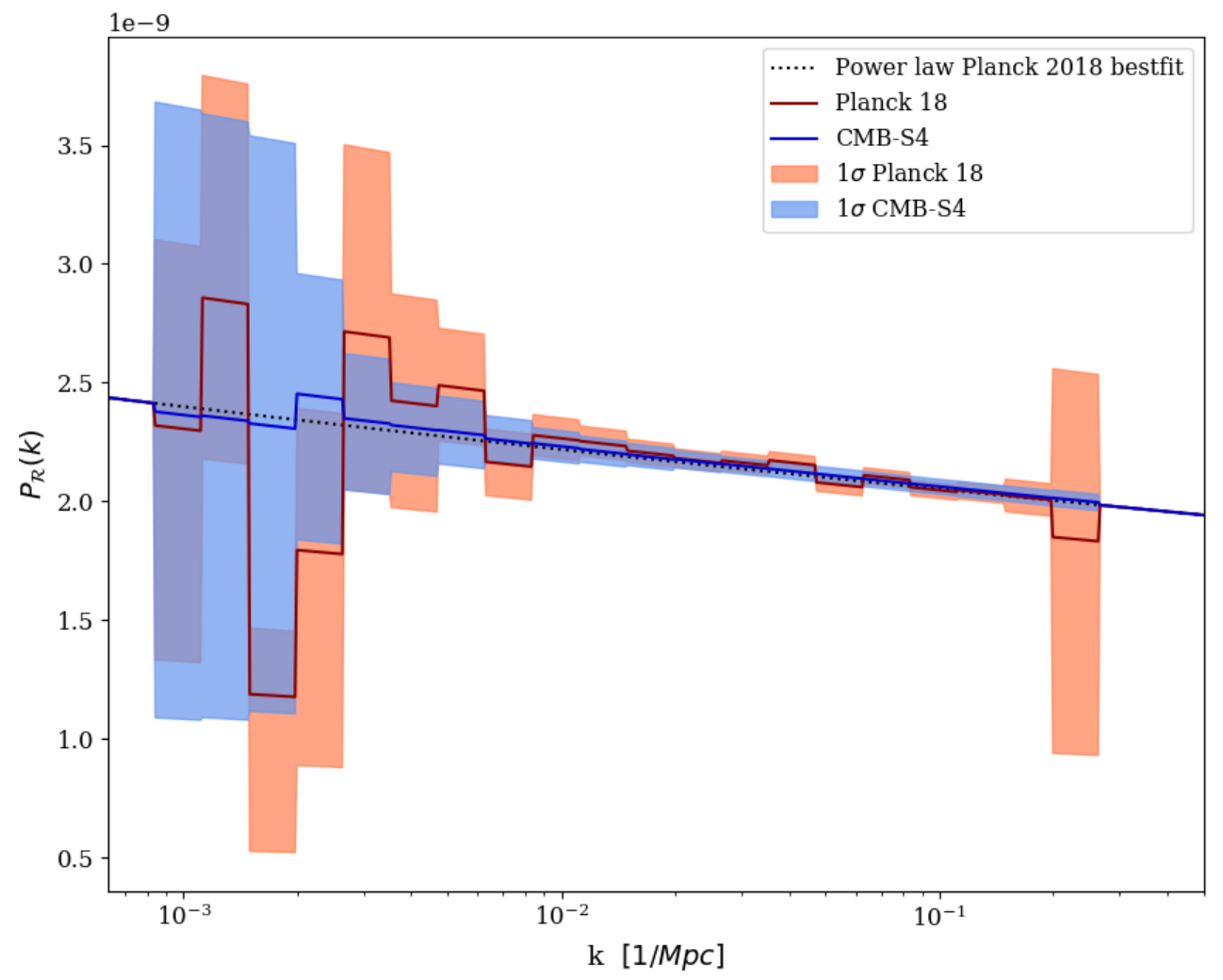

FIG. 1. Reconstructed primordial power spectrum using data from Planck18, with the mean reconstruction shown by the red line and $68 \%$ CL uncertainty band in orange, and projected for CMB-S4 around the best fit Planck18 $\Lambda$ CDM cosmology (dotted black line), with the mean reconstruction shown by the blue line and $68 \%$ CL uncertainty band in blue. Note the CMB-S4 mock data does not include data for $\ell<30$, giving larger uncertainties at the smallest $k$. 


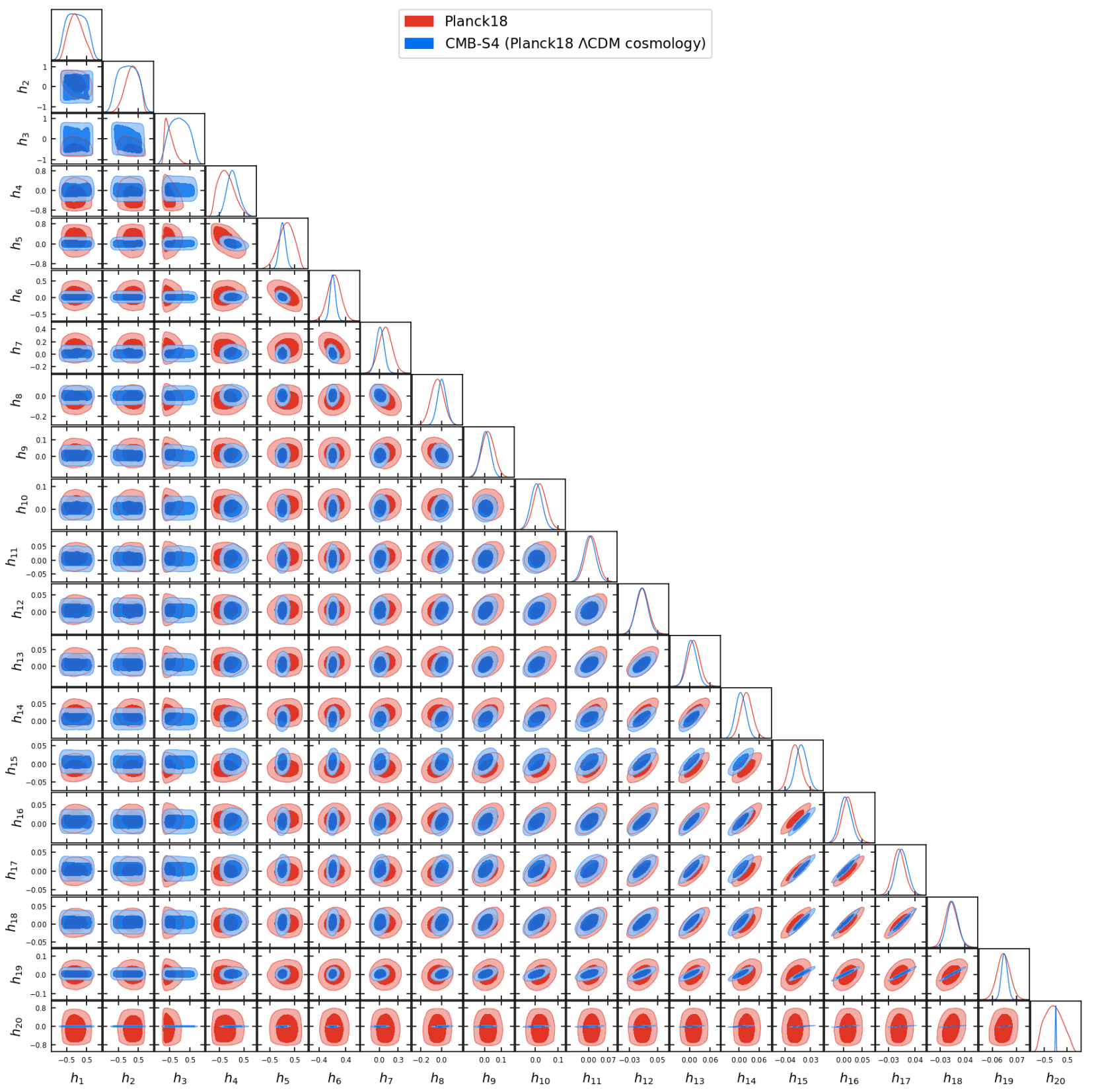

FIG. 2. Corner plot of constraints on the primordial power spectrum amplitudes $h_{i}$ in 20 bins of wavenumber $k$ plotted using data from Planck18 (red), and projected for CMB-S4 (blue). Contours are at $68.3 \%$ and 95.4\% confidence levels, and the marginalized 1D probability distributions are shown as well. 


\begin{tabular}{|c|c|c|c|c|c|c|c|c|}
\hline Param & best-fit & mean $\pm \sigma$ & $95 \%$ lower & $95 \%$ upper & best-fit & mean $\pm \sigma$ & $95 \%$ lower & $95 \%$ upper \\
\hline $100 \Omega_{b} h^{2}$ & 2.228 & $2.222_{-0.02}^{+0.019}$ & 2.183 & 2.26 & 2.237 & $2.238_{-0.0041}^{+0.004}$ & 2.229 & 2.246 \\
\hline$\Omega_{c d m} h^{2}$ & 0.1225 & $0.1222_{-0.0015}^{+0.0015}$ & 0.1191 & 0.1253 & 0.1205 & $0.1202_{-0.00093}^{+0.00084}$ & 0.1184 & 0.122 \\
\hline$H_{0}$ & 66.59 & $65.52_{-1.2}^{+1.9}$ & 62.38 & 68.26 & 67.04 & $67.11_{-0.57}^{+0.79}$ & 65.78 & 68.31 \\
\hline$\tau_{\text {reio }}$ & 0.04342 & $0.05441_{-0.0084}^{+0.0074}$ & 0.03828 & 0.07122 & 0.05387 & $0.05692_{-0.0081}^{+0.008}$ & 0.041 & 0.07327 \\
\hline$\sum m_{\nu}$ & 0.04126 & $0.1747_{-0.17}^{+0.044}$ & $8.011 \times 10^{-6}$ & 0.4507 & 0.07551 & $0.07976_{-0.064}^{+0.038}$ & $2.101 \times 10^{-6}$ & 0.1637 \\
\hline$\sigma_{8}$ & 0.8037 & $0.7908_{-0.027}^{+0.036}$ & 0.7259 & 0.8512 & 0.8084 & $0.8087_{-0.0067}^{+0.0091}$ & 0.7935 & 0.8226 \\
\hline$\Omega_{m}$ & 0.3276 & $0.3418_{-0.015}^{+0.027}$ & 0.305 & 0.3857 & 0.3197 & $0.3186_{-0.0075}^{+0.011}$ & 0.303 & 0.3359 \\
\hline$h_{1}$ & -0.3354 & $-0.03881_{-0.41}^{+0.34}$ & -0.6922 & 0.6651 & 0.07525 & $-0.0108_{-0.45}^{+0.45}$ & -0.758 & 0.754 \\
\hline$h_{2}$ & 0.3522 & $0.1958_{-0.3}^{+0.39}$ & -0.3862 & 0.8 & -0.02108 & $-0.0141_{-0.45}^{+0.45}$ & -0.761 & 0.752 \\
\hline$h_{3}$ & -0.5113 & $-0.4977_{-0.3}^{+0.076}$ & -0.8 & -0.02429 & 0.2763 & $-0.02202_{-0.44}^{+0.44}$ & -0.755 & 0.748 \\
\hline$h_{4}$ & -0.2626 & $-0.2339_{-0.4}^{+0.26}$ & -0.8 & 0.3532 & -0.02197 & $0.04807_{-0.28}^{+0.22}$ & -0.424 & 0.5605 \\
\hline$h_{5}$ & 0.2272 & $0.1701_{-0.29}^{+0.35}$ & -0.4029 & 0.7672 & 0.0342 & $0.01304_{-0.14}^{+0.12}$ & -0.2403 & 0.2758 \\
\hline$h_{6}$ & 0.0392 & $0.05489_{-0.2}^{+0.2}$ & -0.3455 & 0.4553 & -0.03092 & $0.01094_{-0.089}^{+0.08}$ & -0.157 & 0.1831 \\
\hline$h_{7}$ & 0.03037 & $0.09374_{-0.11}^{+0.11}$ & -0.1193 & 0.3067 & -0.02318 & $0.00861_{-0.066}^{+0.061}$ & -0.1185 & 0.1364 \\
\hline$h_{8}$ & -0.04971 & $-0.03859_{-0.065}^{+0.063}$ & -0.1667 & 0.08929 & -0.01839 & $0.005279_{-0.046}^{+0.044}$ & -0.08373 & 0.097 \\
\hline$h_{9}$ & -0.0146 & $0.02106_{-0.04}^{+0.039}$ & -0.05855 & 0.1006 & 0.009427 & $0.005752_{-0.031}^{+0.031}$ & -0.0553 & 0.06685 \\
\hline$h_{10}$ & 0.005821 & $0.01999_{-0.03}^{+0.029}$ & -0.03853 & 0.07943 & -0.00317 & $0.004445_{-0.027}^{+0.025}$ & -0.04639 & 0.05584 \\
\hline$h_{11}$ & -0.0152 & $0.01126_{-0.023}^{+0.022}$ & -0.03422 & 0.05717 & -0.01732 & $0.003982_{-0.022}^{+0.022}$ & -0.0401 & 0.04701 \\
\hline$h_{12}$ & -0.008292 & $0.006973_{-0.019}^{+0.018}$ & -0.03015 & 0.04536 & 0.007063 & $0.005671_{-0.017}^{+0.017}$ & -0.02803 & 0.03938 \\
\hline$h_{13}$ & -0.01717 & $0.01232_{-0.019}^{+0.018}$ & -0.02518 & 0.04983 & 0.004557 & $0.006046_{-0.017}^{+0.016}$ & -0.02522 & 0.03754 \\
\hline$h_{14}$ & 0.001468 & $0.02269_{-0.019}^{+0.018}$ & -0.01407 & 0.05999 & -0.004285 & $0.005611_{-0.017}^{+0.015}$ & -0.02527 & 0.03766 \\
\hline$h_{15}$ & -0.03524 & $-0.01177_{-0.018}^{+0.016}$ & -0.04632 & 0.02338 & 0.001419 & $0.005827_{-0.016}^{+0.016}$ & -0.02549 & 0.03774 \\
\hline$h_{16}$ & -0.008964 & $0.01256_{-0.018}^{+0.016}$ & -0.02156 & 0.04747 & -0.0004784 & $0.005597_{-0.016}^{+0.015}$ & -0.0256 & 0.03694 \\
\hline$h_{17}$ & -0.02743 & $-0.002141_{-0.018}^{+0.015}$ & -0.0354 & 0.0323 & -0.0004859 & $0.005503_{-0.016}^{+0.015}$ & -0.02561 & 0.03713 \\
\hline$h_{18}$ & -0.02281 & $0.002186_{-0.019}^{+0.017}$ & -0.03433 & 0.03931 & -0.0007732 & $0.005426_{-0.016}^{+0.016}$ & -0.02637 & 0.0374 \\
\hline$h_{19}$ & -0.02507 & $0.0008731_{-0.035}^{+0.034}$ & -0.06837 & 0.07003 & -0.0007021 & $0.005276_{-0.017}^{+0.016}$ & -0.0266 & 0.03778 \\
\hline$h_{20}$ & -0.2821 & $-0.07702_{-0.46}^{+0.37}$ & -0.8 & 0.5931 & -0.0007574 & $0.005613_{-0.018}^{+0.017}$ & -0.02914 & 0.04098 \\
\hline
\end{tabular}

TABLE I. Cosmological parameters and the primordial power spectrum amplitudes $h_{i}$ in the 20 bins in wavenumber $k$, for Planck18 data (left columns) and for CMB-S4 mock data generated from the Planck18 $\Lambda$ CDM cosmology (right columns). Param gives the parameter, and 95\% lower and upper give the $95.4 \%$ confidence level lower and upper bounds on the parameter. Nuisance parameters not shown are marginalized over.

While there is some covariance between the $h_{i}$, choosing 20 bins gives a happy medium where features - if they exist - could be well resolved but covariances are not excessive. This will become especially true when we combine CMB and galaxy data. We see no significant evidence for oscillatory features (e.g. low then high values of $h_{i}$ ) or steps, other than the well known dip around $k \approx 2 \times 10^{-3} \mathrm{Mpc}^{-1}$.

Moving to the projections for the future CMB-S4 experiment, shown also in Figs. 1 and 2 and the right hand set of columns of Table I, the PPS is properly consistent with the input power law mock data, and we see significant improvements to the PPS amplitude uncertainties. Beyond the third bin (i.e. $\ell \gtrsim 30$ ) CMB-S4 demonstrates significant improvements over Planck, all the way to the highest $k$ bin, due to its better resolution and noise levels. Half of the amplitudes $1+h_{i}$ are determined to better than $\sim 2 \%$. This will make CMB-S4 a powerful probe of the primordial power spectrum and any features from the inflation physics. (Note we do not include any Planck data in the CMB-S4 case, so there is no $\ell<30$ data and for the first three $k$ bins the constraints are weaker than Planck.)

The cosmology parameters (recall this is for free PPS) mostly follow the usual behaviors from power law PPS. Slight shifts in parameter values between Planck18 and CMB-S4 data generated from Planck18 cosmology can occur since Planck18 is data while CMB-S4 is mock data realized from a $\Lambda$ CDM input cosmology, and since our CMB-S4 realization lacks $\ell<30$ data (just as Planck18 and Planck18 minus low $\ell$ data could give slightly different parameter fits). These shifts are found to be negligible, at most less than the 0.4 sigma level, and so not statistically significant. The cosmological parameter uncertainties with just Planck18 are comparable to those from the standard power law 
case, with the main difference being a factor of roughly two increase in uncertainties on $\sum m_{\nu}, H_{0}$, and $\sigma_{8}$ due to the extra freedom in the PPS amplitude with scale.

The more important aspect is the improvement in parameter uncertainties with future data. Articles such as $[32,33]$ have shown the great advances in cosmological parameter determination enabled by CMB-S4; we find this holds when using free PPS as well. Confidence contours for the cosmology parameters are shown in Fig. 3, making clear the improvement. Since neutrino mass is one of the key aspects of this paper, we note that CMB-S4 reduces the uncertainty on the sum of the neutrino masses from a 95\% CL upper limit of $\sum m_{\nu} \lesssim 0.45 \mathrm{eV}$ to $0.16 \mathrm{eV}$. In the "growth cosmology" plane of $\sum m_{\nu}-\sigma_{8}$ the reduction in uncertainty contour area is more than a factor of 10 . We discuss neutrino mass constraints in greater detail in Sec. IV D.

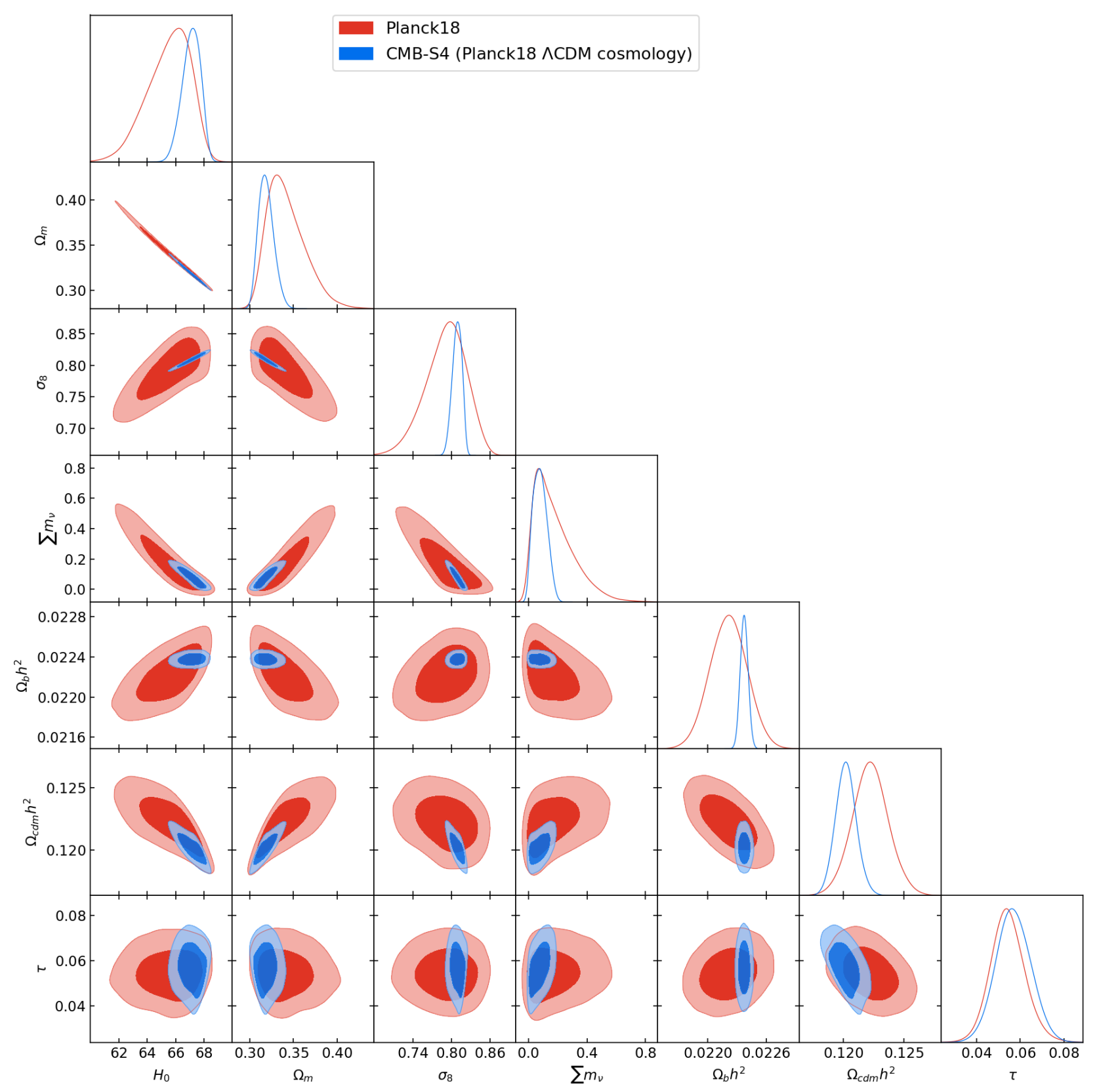

FIG. 3. Corner plot of constraints on the cosmology parameters, including derived ones, are plotted using data from Planck18 (red), and projected for CMB-S4 (blue). CMB-S4 represents a consider tightening of cosmological constraints. 


\section{B. CMB plus Galaxy Surveys}

The combination of the CMB as a probe of the primordial universe with the capability of galaxy surveys to deliver a tomographic view of the evolution of structure formation is especially powerful when the PPS is allowed to be free rather than locked into power law scale dependence.

We now consider the far future case of a high precision CMB experiment (CMB-S4) with a high precision galaxy survey capable of superb tomography over $z=2-5$ (MegaMapper Ideal). This gives enhanced sensitivity to scale dependent physics, such as the PPS and neutrino mass. Results are shown in Table II. The amplitudes $1+h_{i}$ now approach the $0.2 \%$ level or even better, especially at higher $k$ due to the excellent deconvolution of the transfer function, i.e. evolution, from the primordial power spectrum. Only at the largest scales, $k \lesssim 0.015 / \mathrm{Mpc}$, does the uncertainty exceed $1 \%$. Such precision will provide deep insight into any features in the inflationary physics. The covariances between $h_{i}$ are shown in Appendix A. (Note that for the CMB-S4 + MegaMapper Ideal case the neutrino mass has fluctuated a bit below that for CMB-S4 only in Table I, meaning less suppression and so the $h_{i}$ tend to be slightly negative though not by more than $1 \sigma$.

\begin{tabular}{|c|c|c|c|c|c|c|c|c|}
\hline Param & best-fit & $\operatorname{mean} \pm \sigma$ & $95 \%$ lower & $95 \%$ upper & best-fit & $\operatorname{mean} \pm \sigma$ & $95 \%$ lower & $95 \%$ upper \\
\hline $100 \Omega_{b} h^{2}$ & 2.245 & $2.241_{-0.013}^{+0.013}$ & 2.215 & 2.268 & 2.225 & $2.225_{-0.0028}^{+0.0028}$ & 2.219 & 2.23 \\
\hline$\Omega_{c d m} h^{2}$ & 0.1196 & $0.12_{-0.0009}^{+0.0005}$ & 0.1188 & 0.1215 & 0.1194 & $0.1196_{-0.00034}^{+0.00032}$ & 0.119 & 0.1202 \\
\hline$H_{0}$ & 67.97 & $67.56_{-0.38}^{+0.79}$ & 66.37 & 68.54 & 67.71 & $67.54_{-0.25}^{+0.25}$ & 67.05 & 68.02 \\
\hline$\tau_{\text {reio }}$ & 0.05391 & $0.05413_{-0.0033}^{+0.0032}$ & 0.04765 & 0.06073 & 0.05497 & $0.05471_{-0.00091}^{+0.00091}$ & 0.05292 & 0.0565 \\
\hline$\sum m_{\nu}$ & 0.01977 & $0.04514_{-0.045}^{+0.013}$ & $8.615 \times 10^{-7}$ & 0.09908 & 0.0368 & $0.04844_{-0.013}^{+0.014}$ & 0.02158 & 0.07562 \\
\hline$\sigma_{8}$ & 0.8187 & $0.8146_{-0.003}^{+0.0045}$ & 0.8074 & 0.8212 & 0.8148 & $0.8123_{-0.0023}^{+0.0022}$ & 0.8079 & 0.8168 \\
\hline$\Omega_{m}$ & 0.3081 & $0.3133_{-0.0043}^{+0.01}$ & 0.3015 & 0.3282 & 0.31 & $0.3123_{-0.0033}^{+0.0034}$ & 0.3062 & 0.3187 \\
\hline$h_{1}$ & -0.0598 & $-0.07303_{-0.38}^{+0.31}$ & -0.7289 & 0.5878 & 0.4595 & $0.006577_{-0.46}^{+0.46}$ & -0.7009 & 0.7636 \\
\hline$h_{2}$ & 0.02348 & $0.01665_{-0.28}^{+0.27}$ & -0.5167 & 0.5634 & -0.1442 & $-0.001408_{-0.39}^{+0.37}$ & -0.6671 & 0.6684 \\
\hline$h_{3}$ & -0.4163 & $-0.3033_{-0.23}^{+0.2}$ & -0.7177 & 0.109 & -0.04378 & $-0.005165_{-0.22}^{+0.21}$ & -0.4426 & 0.4387 \\
\hline$h_{4}$ & -0.1129 & $-0.0614_{-0.15}^{+0.14}$ & -0.3462 & 0.2266 & -0.07495 & $-0.003495_{-0.13}^{+0.12}$ & -0.2522 & 0.2465 \\
\hline$h_{5}$ & -0.06181 & $0.0138_{-0.092}^{+0.092}$ & -0.1704 & 0.199 & -0.04954 & $-0.003597_{-0}^{+0}$ & -0.1413 & 0.1378 \\
\hline$h_{6}$ & 0.002678 & $0.01869_{-0.057}^{+0.055}$ & -0.09206 & 0.1325 & -0.02603 & $-0.006101_{-0}^{+0}$ & -0.09166 & 0.07955 \\
\hline$h_{7}$ & 0.007194 & $0.007343_{-0.034}^{+0.035}$ & -0.0618 & 0.07659 & 0.001492 & $-0.005252_{-0.029}^{+0.029}$ & -0.06131 & 0.05167 \\
\hline$h_{8}$ & 0.001145 & $-0.007705_{-0.03}^{+0.029}$ & -0.06656 & 0.05261 & 0.004995 & $-0.006739_{-0.024}^{+0.023}$ & -0.05306 & 0.04054 \\
\hline$h_{9}$ & 0.004989 & $0.008361_{-0.032}^{+0.031}$ & -0.05434 & 0.07157 & -0.02778 & $-0.001105_{-0.026}^{+0.026}$ & -0.05245 & 0.05036 \\
\hline$h_{10}$ & 0.001783 & $0.001536_{-0.015}^{+0.014}$ & -0.02684 & 0.03112 & -0.003038 & $-0.00514_{-0.011}^{+0.01}$ & -0.02642 & 0.01609 \\
\hline$h_{11}$ & 0.001936 & $-0.0004983_{-0.013}^{+0.011}$ & -0.02376 & 0.0246 & -0.004895 & $-0.003653_{-0.0081}^{+0.0084}$ & -0.02024 & 0.01292 \\
\hline$h_{12}$ & -0.003505 & $-0.001884_{-0.011}^{+0.0088}$ & -0.02081 & 0.01824 & -0.002216 & $-0.002861_{-0.0056}^{+0.0057}$ & -0.01436 & 0.008479 \\
\hline$h_{13}$ & -0.004738 & $0.0003305_{-0.0092}^{+0.0071}$ & -0.01509 & 0.01701 & -0.001704 & $-0.002775_{-0.0041}^{+0.0041}$ & -0.01113 & 0.005437 \\
\hline$h_{14}$ & -0.0003906 & $0.0005026_{-0.0084}^{+0.0064}$ & -0.0135 & 0.01575 & -0.002986 & $-0.003812_{-0.0034}^{+0.0034}$ & -0.01053 & 0.002928 \\
\hline$h_{15}$ & -0.002715 & $-0.001394_{-0.0073}^{+0.0054}$ & -0.01344 & 0.01167 & -0.0031 & $-0.002622_{-0.0027}^{+0.0026}$ & -0.007802 & 0.002705 \\
\hline$h_{16}$ & -0.001334 & $0.000247_{-0.0049}^{+0.0041}$ & -0.008609 & 0.009371 & -0.001665 & $-0.002109_{-0.0021}^{+0.0021}$ & -0.006296 & 0.002054 \\
\hline$h_{17}$ & -0.002041 & $-0.0002224_{-0.0048}^{+0.0038}$ & -0.008547 & 0.008522 & -0.0006202 & $-0.001137_{-0.0019}^{+0.0019}$ & -0.004865 & 0.002604 \\
\hline$h_{18}$ & -0.0008104 & $0.0002547_{-0.0041}^{+0.0035}$ & -0.007308 & 0.00795 & -0.001353 & $-0.001856_{-0.0018}^{+0.0017}$ & -0.005318 & 0.001608 \\
\hline$h_{19}$ & -0.0001978 & $0.0002558_{-0.0029}^{+0.0026}$ & -0.005191 & 0.005824 & -0.000699 & $-0.001001_{-0.0014}^{+0.0014}$ & -0.003771 & 0.001864 \\
\hline$h_{20}$ & -0.0008644 & $0.00001845_{-0.0015}^{+0.0015}$ & -0.003015 & 0.003055 & 0.0007476 & $0.0001666_{-0.001}^{+0.001}$ & -0.00184 & 0.002188 \\
\hline
\end{tabular}

TABLE II. Cosmological parameters and the primordial power spectrum amplitudes $h_{i}$ in the 20 bins in wavenumber $k$, for Planck18 plus MegaMapper Fiducial mock data (left columns) and for CMB-S4 plus MegaMapper Ideal mock data generated from the best fit Planck18 $\Lambda$ CDM cosmology (right columns).

Neutrino mass constraints improve to the $\sigma\left(\sum m_{\nu}\right) \approx 0.014 \mathrm{eV}$ level, allowing a clear detection of the minimal mass sum according to neutrino oscillation experiments and potentially significant distinction between the normal and inverted mass hierarchies. Most other cosmological parameters show similar improvements in precision, of order 
a factor 3-4. The cosmology parameter constraints are shown in Fig. 4. The shift in $\Omega_{b} h^{2}$ upon adding galaxy data can be traced back to interaction between the free PPS and the nonlinear correction parameters $S_{i}$ (and the knock-on effects on other parameters). The astrophysical parameters $b_{i}$ and $S_{i}$ are also well constrained (see Appendix A).

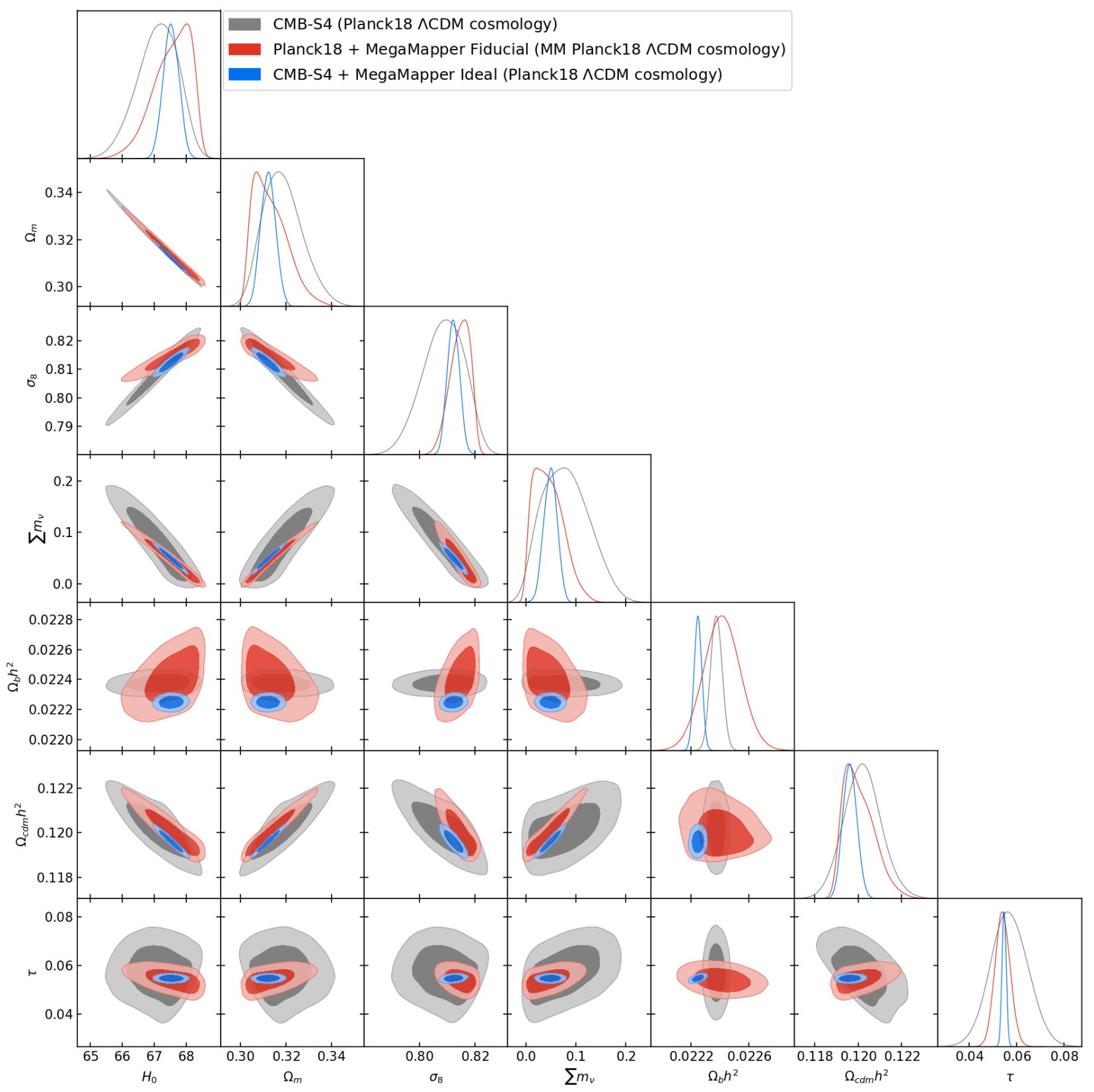

FIG. 4. Corner plot of constraints on the cosmology parameters, included derived ones, are plotted using data from CMB-S4 (grey; as in Fig. 3), Planck18 plus MegaMapper Fiducial (red), and CMB-S4 plus MegaMapper Ideal (blue).

Perhaps most unusual is the effect on the reionization optical depth $\tau$. In the standard power law PPS case the optical depth is degenerate with the amplitude of the primordial curvature perturbation power $A_{s}$ in the form $A_{s} e^{-2 \tau}$. In our free binned version of the PPS, due to the freedom allowed by the 20 amplitude bins, we can independently probe large and small scales of the PPS. In combining CMB and tomographic galaxy clustering one achieves excellent $\sigma\left(h_{i}\right)$ at small scales, and these are translated into tight constraints on $\sigma(\tau)$; by contrast, in the power law case we cannot separate large from small scales in the PPS amplitude. This holds as well with CMB only data, where constraints on $\tau$ match well projections in the literature, and even for Planck18 plus MegaMapper Fiducial (though we start to see some improvement there). The separation of scales becomes especially effective at the high redshifts of 
MegaMapper where smaller scales stay more linear, and most so for MegaMapper Ideal with its higher number density, plus CMB-S4 with its better resolution and noise. Figure 5 illustrates the 1D posterior distributions of the reionization optical depth in the CMB-S4 + MegaMapper Ideal case (and also CMB-S4 alone) for the power law and free binned PPS cases. Comparing the blue curve with the black demonstrates the role of future galaxy clustering data, while keeping the same binned PPS model. Comparing the blue curve with the green - i.e. fixing to the CMB+galaxy data sets - demonstrates that using binned PPS instead of power law PPS is what gives the improved constraint on tau, as discussed above.

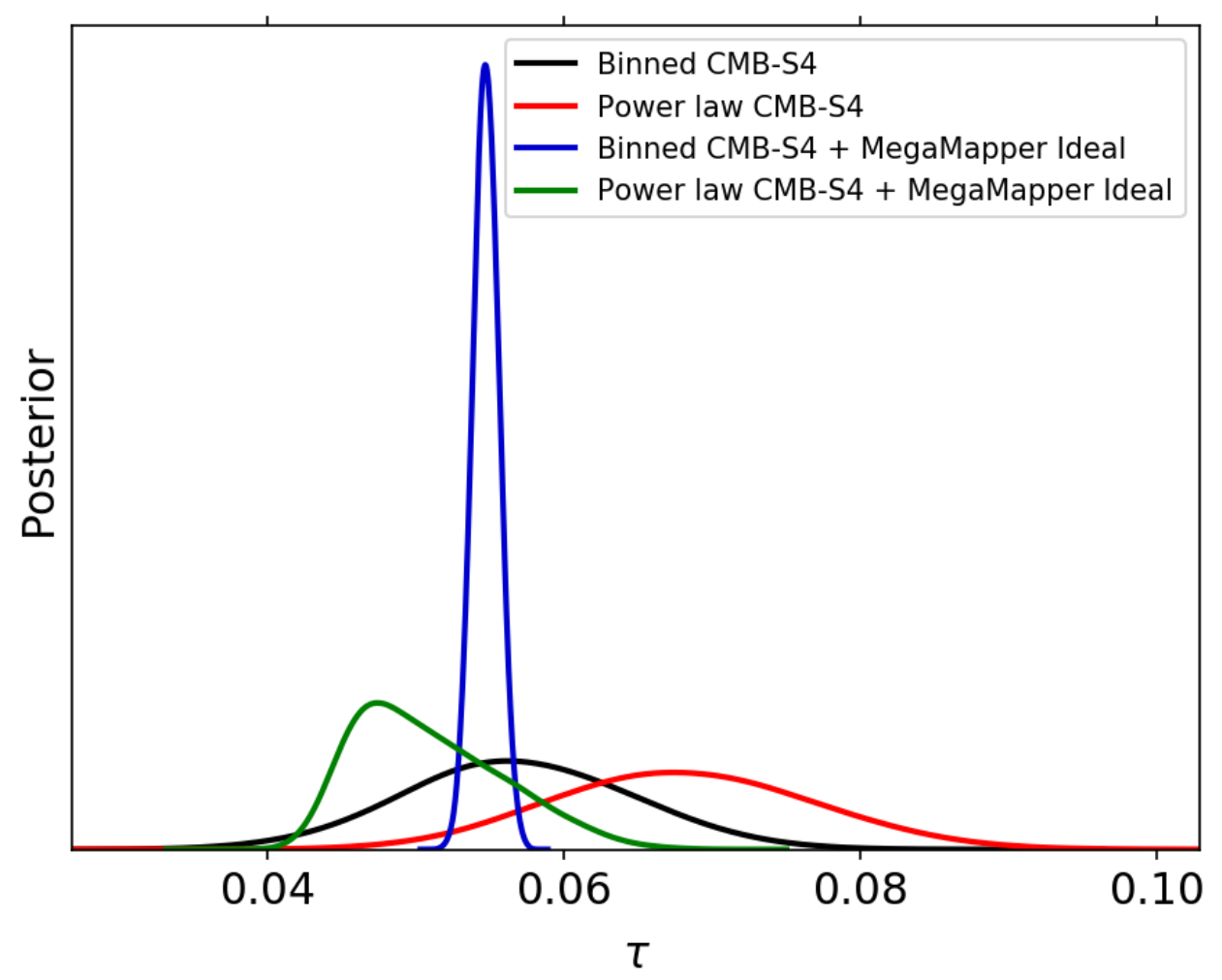

FIG. 5. 1D PDFs of the reionization optical depth $\tau$ are contrasted for the free, binned PPS and the standard restriction to power law PPS, for the cases of CMB-S4 alone and CMB-S4 plus MegaMapper Ideal. As discussed in the text, the highly peaked and tightly distributed form of the posterior for the binned version is a manifestation of the decoupling of short and large scales due to the free amplitudes in $k$ bins, which can be taken advantage of by the multiple redshift bins in the galaxy data to deconvolve the primordial conditions from the transfer function. The same behavior is not present in the power law PPS, as we have only one degree of freedom when constraining the amplitude, i.e. shifting the PPS up or down as a whole.

In the nearer future is the case using the current CMB data from Planck18 and projections from the less ambitious MegaMapper Fiducial. This delivers quite respectable constraints on the PPS, a factor of a few better on $h_{i}$ at the higher $k$ than without galaxy data, though about a factor 2 worse than the CMB-S4 + MegaMapper Ideal case. The cosmology constraints for this case are also shown in Table II and Fig. 4. Again we see that in the "growth" plane of $\sum m_{\nu}-\sigma_{8}$, the combination of CMB-S4 + MegaMapper Ideal improves by a further factor of 10 relative to CMB-S4 alone, and by a factor of several on Planck18 + MegaMapper Fiducial. We compare the full cosmology parameter results between the free PPS and power law PPS for CMB-S4 + MegaMapper Ideal and for Planck18 + MegaMapper Fiducial in Appendix B.

\section{Summary of Primordial Power Spectrum Constraints}

For inflation physics we see that the upcoming generation of CMB and large scale structure experiments can deliver strong improvements over the current constraints on the primordial curvature power spectrum. This will be highly 
useful in exploring for properties in inflation such as bends or steps in the inflationary potential or additional fields that would have signatures in oscillations or sharp features in the primordial power spectrum. Freedom beyond a power law scale dependence would be an important discovery and clue to high energy physics.

Figure 6 shows the constraints enabled by the next generation of experiments. Note this does not account for improvements in the low multipoles $\ell<30$; polarization data from satellite missions such as LiteBIRD [40, 41] (and possibly ground based experiments with sufficient systematics control) could lead to further gains there. Note that large scale structure data plays a significant role for $k \gtrsim$ few $\times 10^{-3} \mathrm{Mpc}^{-1}$ even with excellent CMB data.

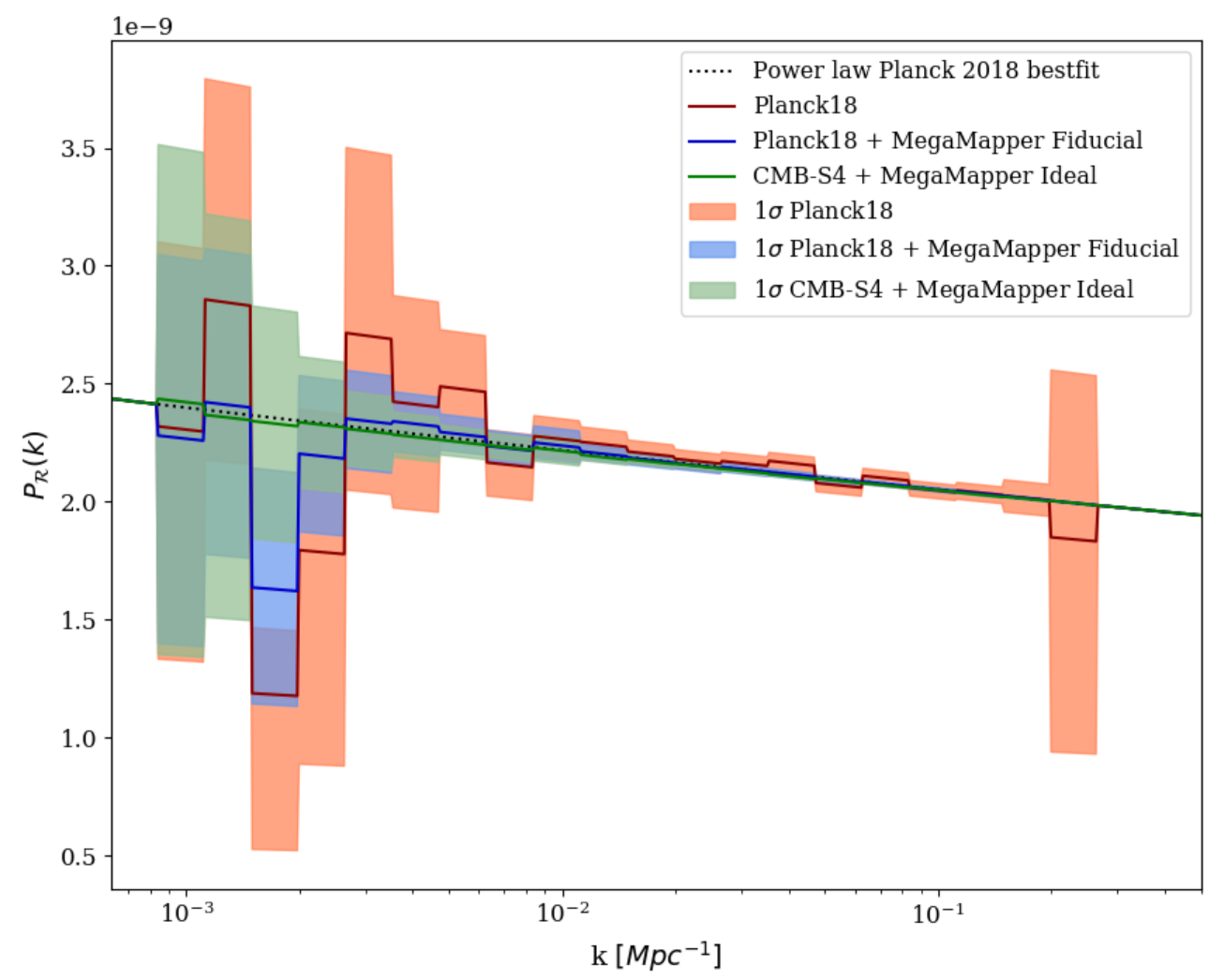

FIG. 6. Reconstructed primordial power spectrum using actual data from Planck18 (red, as in Fig. 1), Planck18 plus MegaMapper Fiducial (blue), and CMB-S4 plus MegaMapper Ideal (green). Future data will be able to significantly narrow in on, or find deviations from, power law behavior in scale.

In order to examine more closely the effects of the upcoming data on primordial power spectrum reconstruction, and the ability to distinguish deviations from a pure power law behavior, Fig. 7 shows the fractional residuals of each reconstruction (including CMB-S4 alone) relative to its best power law fit, for $k \geq 0.01 \mathrm{Mpc}^{-1}$ to allow a zoomed in scale. The improvements with each successive data set are clear.

\section{Summary of Neutrino Mass Constraints}

For neutrino physics in the form of the sum of the neutrino masses (and its implications for the neutrino hierarchy), large scale structure surveys offer great complementarity with CMB experiments. While CMB-S4 can reduce the uncertainty from the $\sim 110 \mathrm{meV}$ of Planck alone to $\sim 50 \mathrm{meV}$, the addition of galaxy redshift survey data can give $\sim 25 \mathrm{meV}$ for Planck plus MegaMapper Fiducial or $\sim 14 \mathrm{meV}$ for CMB-S4 plus MegaMapper Ideal. These constraints are not substantially different from those obtained in the pure power law case without inflationary freedom, showing that the combination of CMB and large scale structure can successfully fit both early and late cosmology simultaneously. 


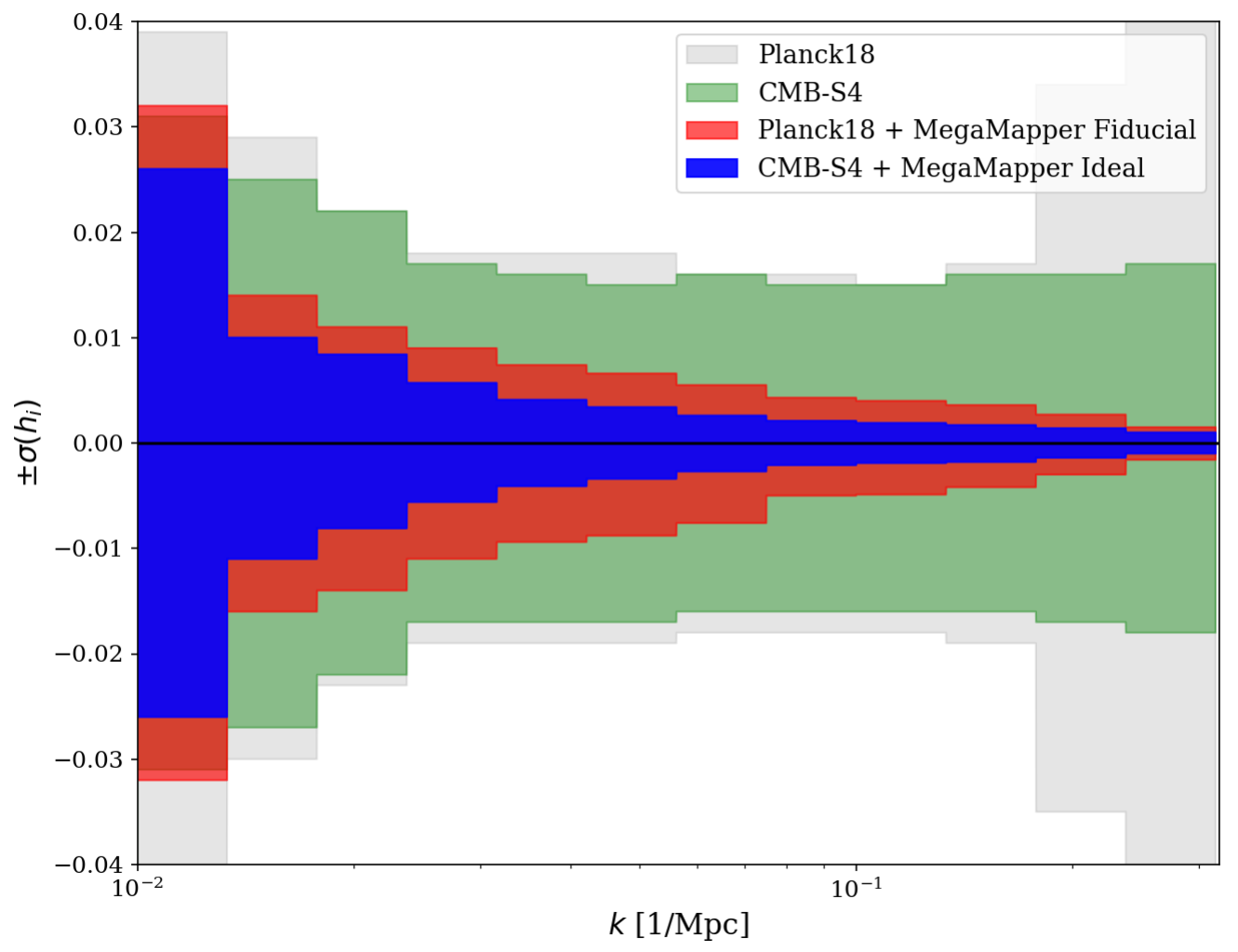

FIG. 7. Fractional residuals of primordial power spectra residuals relative to their best fit power laws, zoomed into the $k>0.01 \mathrm{Mpc}^{-1}$ range. We see each next generation of experiments provides significant improvements in constraining power, with mean precisions over this range of $5.5 \%$ for Planck18 current data (grey), 1.8\% for CMB-S4 (green), $0.83 \%$ for Planck18 plus MegaMapper Fiducial (red), to 0.57\% for CMB-S4 plus MegaMapper Ideal (blue).

Figure 8 shows the 1D PDF for the sum of neutrino masses in these four cases.

\section{CONCLUSIONS}

The very high energy early universe and the physics of inflation are outstanding frontiers to explore. Although the simplest theories predict a power law form for the primordial power spectrum of curvature perturbations, considerable freedom and diverse possibilities exist. We allow for inflationary freedom and show that sufficiently precise CMB measurements over a range of angular scales and large scale structure surveys in the late universe over a range of redshifts can not only accommodate such freedom but return substantially similar cosmological parameter estimation as the restrictive power law assumption. That is, future data will allow precise constraints on both the early and late universe simultaneously.

CMB and galaxy redshift surveys work extremely well together, and we quantify, by probe or combination of probes, and by generation of experiment, how well they can reconstruct the primordial power spectrum and late time fundamental parameters such as the sum of neutrino masses.

We reconstruct the primordial power spectrum in a free form of 20 bins in wavenumber, allowing stringent tests of the power law behavior or revealing features such as steps, bends, or oscillations from inflation physics. Due to the power of deconvolution of initial conditions and late time transfer functions due to the multiple bins in angular scale and redshift of the data sets, the free bin amplitudes can be constrained with subpercent precisions for $k \gtrsim 0.015 \mathrm{Mpc}^{-1}$. Indeed, some bins can reach $0.1 \%$ precision and for the further future combination CMB-S4 plus MegaMapper Ideal the average precision over the range $k=0.01-0.35 \mathrm{Mpc}^{-1}$ can reach $0.6 \%$. 


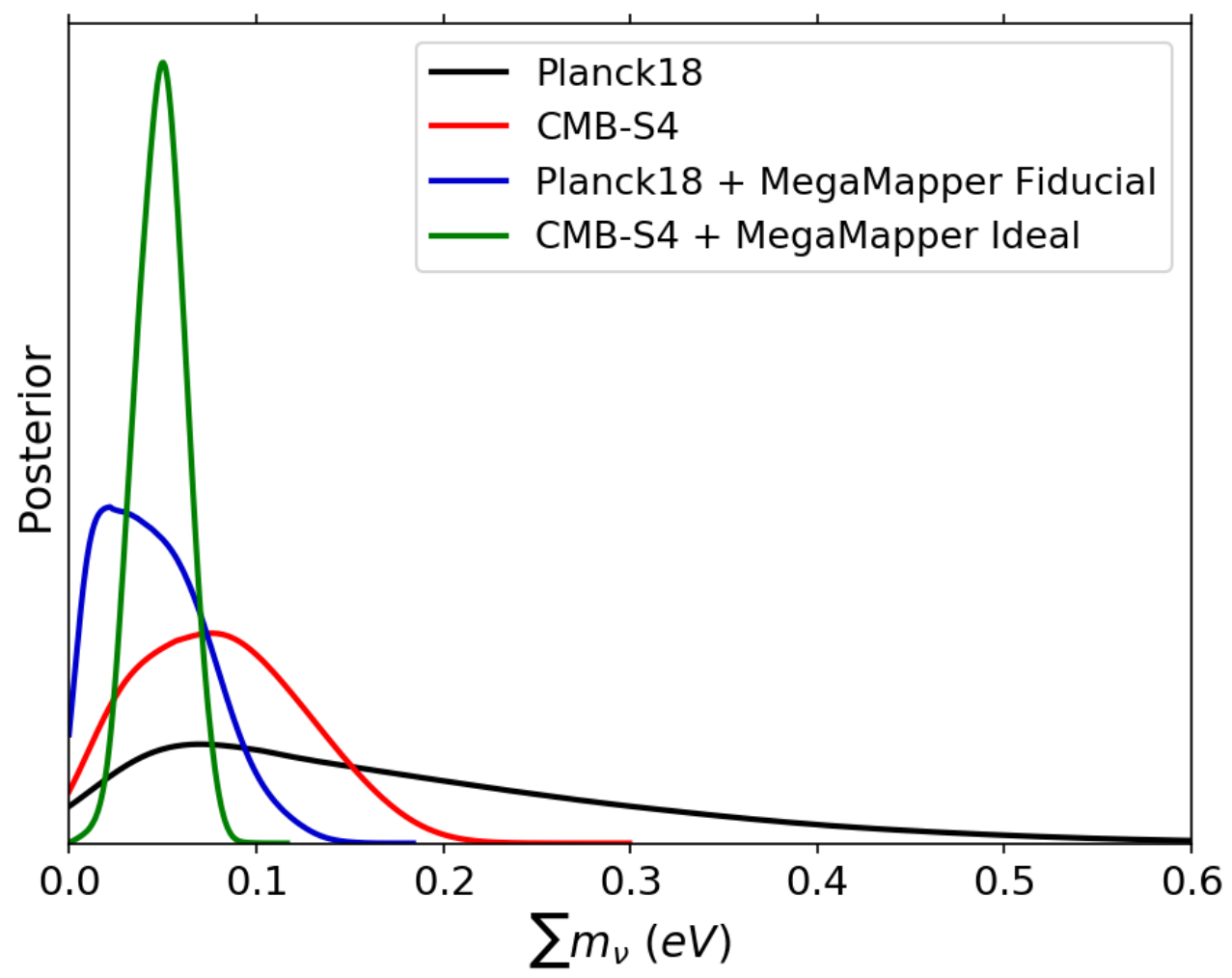

FIG. 8. 1D PDFs for the sum of neutrino masses are shown for actual data from Planck18, and projected for CMB-S4 alone, Planck18 plus MegaMapper Fiducial, and CMB-S4 plus MegaMapper Ideal. Each more advanced experiment continues improving the constraints, with CMB-S4 plus MegaMapper Ideal capable of clearly ruling out both zero neutrino mass and the inverted hierarchy.

The $95 \%$ confidence upper limit on the sum of neutrino masses - even allowing for inflationary freedom - will drop from $0.45 \mathrm{eV}$ with current Planck18 data to $0.16 \mathrm{eV}$ with CMB-S4 (assuming minimum mass). The $68 \%$ confidence level uncertainty will drop from $110 \mathrm{meV}$ to $50 \mathrm{meV}$, and with the addition of MegaMapper Ideal it can reach 14 meV. This would allow a stringent test of neutrino oscillation bounds on the minimum sum of masses, and possible distinction between the normal and inverted hierarchies. An advantage of MegaMapper is its high redshift range, $z \gtrsim 2$, with a huge volume encompassing more modes than measured in the CMB, and increased wavenumber range in the linear regime. That both makes interpretation of data easier and gives a longer lever arm for discernment of scale dependent neutrino mass effects. Furthermore we expect the universe to be mostly matter dominated there, with reduced dependence on dark energy properties.

If one considers the "growth" plane of $\sum m_{\nu}-\sigma_{8}$, the progress from Planck18 to CMB-S4 delivers a factor 10 improvement in the confidence contour area, and adding MegaMapper Ideal provides a further factor 10 beyond that.

Further improvements are possible by including the multipoles $\ell<30$ we have left out, either from present data or future experiments such as LiteBIRD, and including large scale structure surveys at $z<2$. The latter can provide strong constraints on dark energy properties beyond $\Lambda$ CDM.

Overall we have seen that freedom can lead to discovery in the early universe, and poses no obstacle to discovery in the late universe (i.e. the error bars are not appreciably worse than those under the power law assumption), with the excellent data sets that the next decade will bring.

\section{ACKNOWLEDGMENTS}

This work made use of the CHE cluster, managed and funded by COSMO/CBPF/MCTI, with financial support from FINEP and FAPERJ, and operating at the Javier Magnin Computing Center/CBPF. This work is supported 
in part by the Energetic Cosmos Laboratory and by the U.S. Department of Energy, Office of Science, Office of High Energy Physics, under Award DE-SC-0007867 and contract no. DE-AC02-05CH11231. GB would like to acknowledge the State Scientific and Innovation Funding Agency of Espirito Santo (FAPES, Brazil). GB would also like to thank the Brazilian Physical Society (SBF) through the SBF/APS PhD Exchange Program for financial support and LBL for the hospitality and financial support during the early stages of this work. This study was financed in part by the Coordenação de Aperfeiçoamento de Pessoal de Nível Superior - Brasil (CAPES) - Finance Code 001.

\section{Appendix A: More Detailed Results}

Here we give additional information concerning the galaxy survey bias and power correction terms, and the PPS bin amplitudes, mostly focused on covariances.

The astrophysics parameters of the galaxy bias $b_{i}$ and power correction $S_{i}$ in each redshift slice of the galaxy redshift survey do not have extreme degeneracies with the cosmology parameters discussed in the main text. For completeness, we show a corner plot of $b_{i}$ and $S_{i}$ against each other in Fig. 9, for the case of Planck18 plus MegaMapper Fiducial (so four redshift slices). This gives a sense for their constraints.

Similarly, the covariances among the PPS amplitudes $h_{i}$ are moderate, especially with the excellent discrimination in wavenumber and redshift of the CMB-S4 plus MegaMapper Ideal surveys. The corner plot is shown in Fig. 10.

\section{Appendix B: Freedom vs Power Law}

For most of the cosmological parameters the combination of the CMB data and galaxy redshift survey data gives strong enough constraints over the range of angular scales and redshifts to enable comparable parameter fitting to the power law case, despite allowing the extra freedom beyond a power law assumption in the PPS. That is, the combined data enables exploration of both early and late cosmology successfully.

Figures 11 and 12 show the comparison between free and power law PPS results for CMB-S4 plus MegaMapper Ideal and Planck18 plus MegaMapper Fiducial respectively. Slights offsets and the behavior of the optical depth $\tau$ were discussed in Sec. IV B.

[1] R. de Putter, E. V. Linder, and A. Mishra, Inflationary Freedom and Cosmological Neutrino Constraints, Phys. Rev. D 89 (2014), 103502, arXiv:1401.7022.

[2] W. H. Kinney, How to fool CMB parameter estimation , Phys. Rev. D 63 (2001), 043001, arXiv:astro-ph/0005410.

[3] D. K. Hazra, A. Shafieloo, and T. Souradeep, Cosmological parameter estimation with free-form primordial power spectrum, Phys.Rev. D87 (2013), 123528, arXiv:1303.5336.

[4] G.-B. Zhao, S. Saito, W. J. Percival, A. J. Ross, F. Montesano, M. Viel, D. P. Schneider, M. Manera, J. Miralda-Escudé, N. Palanque-Delabrouille, et al., The clustering of galaxies in the SDSS-III Baryon Oscillation Spectroscopic Survey: weighing the neutrino mass using the galaxy power spectrum of the CMASS sample, Mon. Not. Roy. Astron. Soc. 436 (2013), 2038-2053, arXiv:1211.3741

[5] S. Hannestad, Reconstructing the inflationary power spectrum from CMBR data, Phys. Rev. D 63 (2001), 043009, arXiv:astro-ph/0009296.

[6] R. Hlozek, J. Dunkley, G. Addison, J. W. Appel, J. R. Bond, C. Sofia Carvalho, S. Das, M. J. Devlin, R. Dünner, T. Essinger-Hileman, et al., The Atacama Cosmology Telescope: a measurement of the primordial power spectrum, Astrophys. J. 749 (2012) 90, arXiv:1105.4887.

[7] C. Gauthier and M. Bucher, Reconstructing the primordial power spectrum from the CMB, JCAP 1210 (2012) 050, arXiv:1209.2147.

[8] E. Di Valentino, S. Gariazzo, M. Gerbino, E. Giusarma, and O. Mena, Dark Radiation and Inflationary Freedom after Planck 2015, Phys. Rev. D 93 (2016), 083523, arXiv:1601.07557.

[9] S. Gariazzo, L. Lopez-Honorez, and O. Mena, Primordial power spectrum features and $f_{\mathrm{NL}}$ constraints, Phys. Rev. D 92 (2015), 063510, arXiv:1506.05251.

[10] A. Ravenni, L. Verde, and A. J. Cuesta, Red, Straight, no bends: primordial power spectrum reconstruction from CMB and large-scale structure, JCAP 1608 (2016) 028, arXiv:1605.06637.

[11] N. Canac, G. Aslanyan, K. N. Abazajian, R. Easther, and L. C. Price, Testing for New Physics: Neutrinos and the Primordial Power Spectrum, JCAP 1609 (2016) 022, arXiv:1606.03057.

[12] P. Hunt and S. Sarkar, Search for features in the spectrum of primordial perturbations using Planck and other datasets, JCAP 1512 (2015) 052, arXiv:1510.03338. 


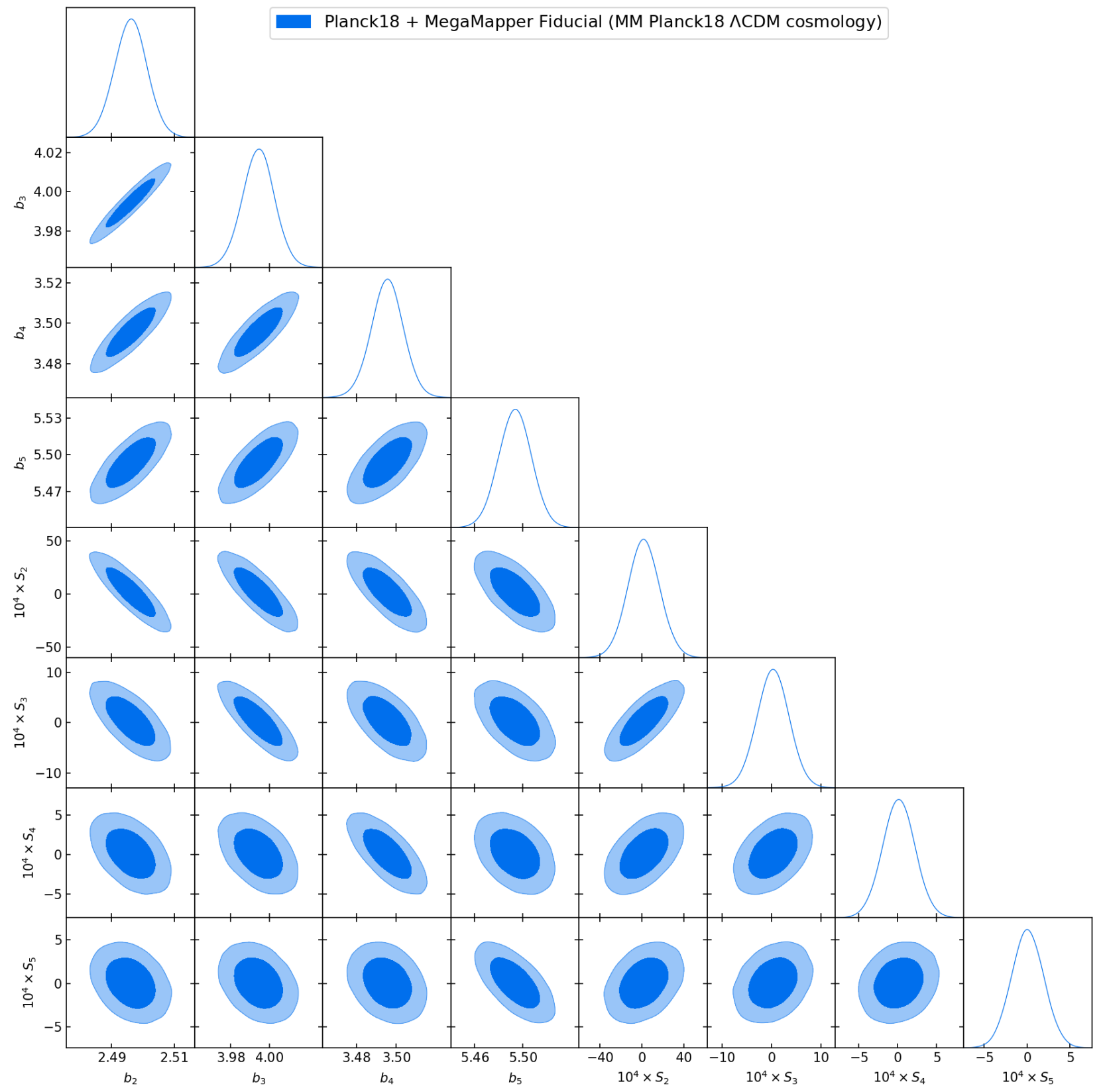

FIG. 9. Corner plot of galaxy bias $b_{i}$ and power correction $S_{i}$ terms in each redshift bin (e.g. $b_{3}$ means the bias in the $z=3$ redshift bin), for the case of Planck18 plus MegaMapper Fiducial.

[13] D. K. Hazra, D. Paoletti, M. Ballardini, F. Finelli, A. Shafieloo, G. F. Smoot, and A. A. Starobinsky, Probing features in inflaton potential and reionization history with future CMB space observations, JCAP 1802 (2018) 017, arXiv:1710.01205.

[14] D. K. Hazra, A. Shafieloo, and T. Souradeep, Parameter discordance in Planck CMB and low redshift measurements: projection in the primordial power spectrum, JCAP 1904 (2019) 036, arXiv:1810.08101.

[15] A. Slosar, X. Chen, C. Dvorkin, D. Green, P. D. Meerburg, E. Silverstein et al., Scratches from the Past: Inflationary Archaeology through Features in the Power Spectrum of Primordial Fluctuations, arXiv:1903.09883.

[16] J. Chluba, J. Hamann and S. P. Patil, Features and New Physical Scales in Primordial Observables: Theory and Observation, Int. J. Mod. Phys. D 24 (2015), 1530023, arXiv:1505.01834.

[17] F. Beutler, M. Biagetti, D. Green, A. Slosar, B. Wallisch, Primordial Features from Linear to Nonlinear Scales, Phys. Rev. Research 1 (2019) 033209, arXiv:1906.08758.

[18] C. Zeng, E. Kovetz, X. Chen, J. Munoz, and M. Kamionkowski, Searching for Oscillations in the Primordial Power Spectrum with CMB and LSS Data, Phys. Rev. D 99 (2019), 043517, arXiv:1812.05105. 


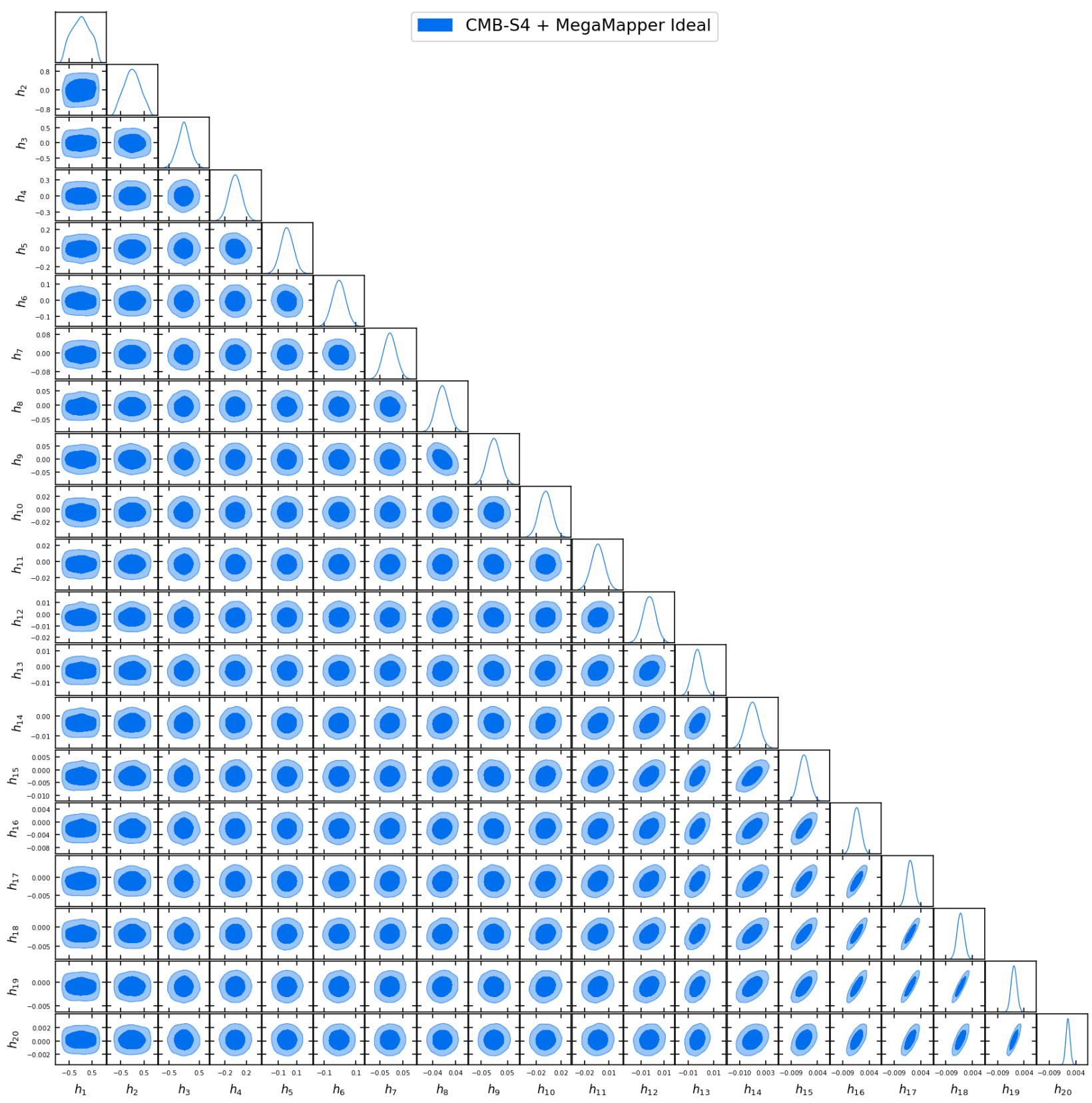

FIG. 10. Corner plot of PPS amplitudes $h_{i}$ in each $k$ bin, for the case of CMB-S4 plus MegaMapper Ideal.

[19] G. Domnech and M. Kamionkowski, Lensing anomaly and oscillations in the primordial power spectrum, JCAP 1911 (2019) 040 arXiv:1905.04323.

[20] W. J. Handley, A. N. Lasenby, S. P. Patil, V. H. Peiris and M. P. Hobson, Bayesian inflationary reconstructions from Planck 2018 data, Phys. Rev. D 100 (2019), 103511, arXiv:1908.00906.

[21] F. Leclercq, W. Enzi, J. Jasche, A. Heavens, Primordial power spectrum and cosmology from black-box galaxy surveys, Mon. Not. Roy. Astron. Soc. 490 (2019), 4237, arXiv:1902.10149.

[22] Simons Observatory Collaboration, The Simons Observatory: Science goals and forecasts, JCAP 1902 (2019) 056, arXiv: 1808.07445.

[23] Planck Collaboration, Planck 2018 results. X. Constraints on inflation, arXiv:1807.06211.

[24] X. Chen, C. Dvorkin, Z. Huang, M. H. Namjoo, and L. Verde, The Future of Primordial Features with Large-Scale Structure Surveys, JCAP 1611 (2016) 014, arXiv:1605.09365.

[25] D. K. Hazra, A. Shafieloo, G. F. Smoot and A. A. Starobinsky, Primordial features and Planck polarization, JCAP 1609 (2016) 009, arXiv:1605.02106. 


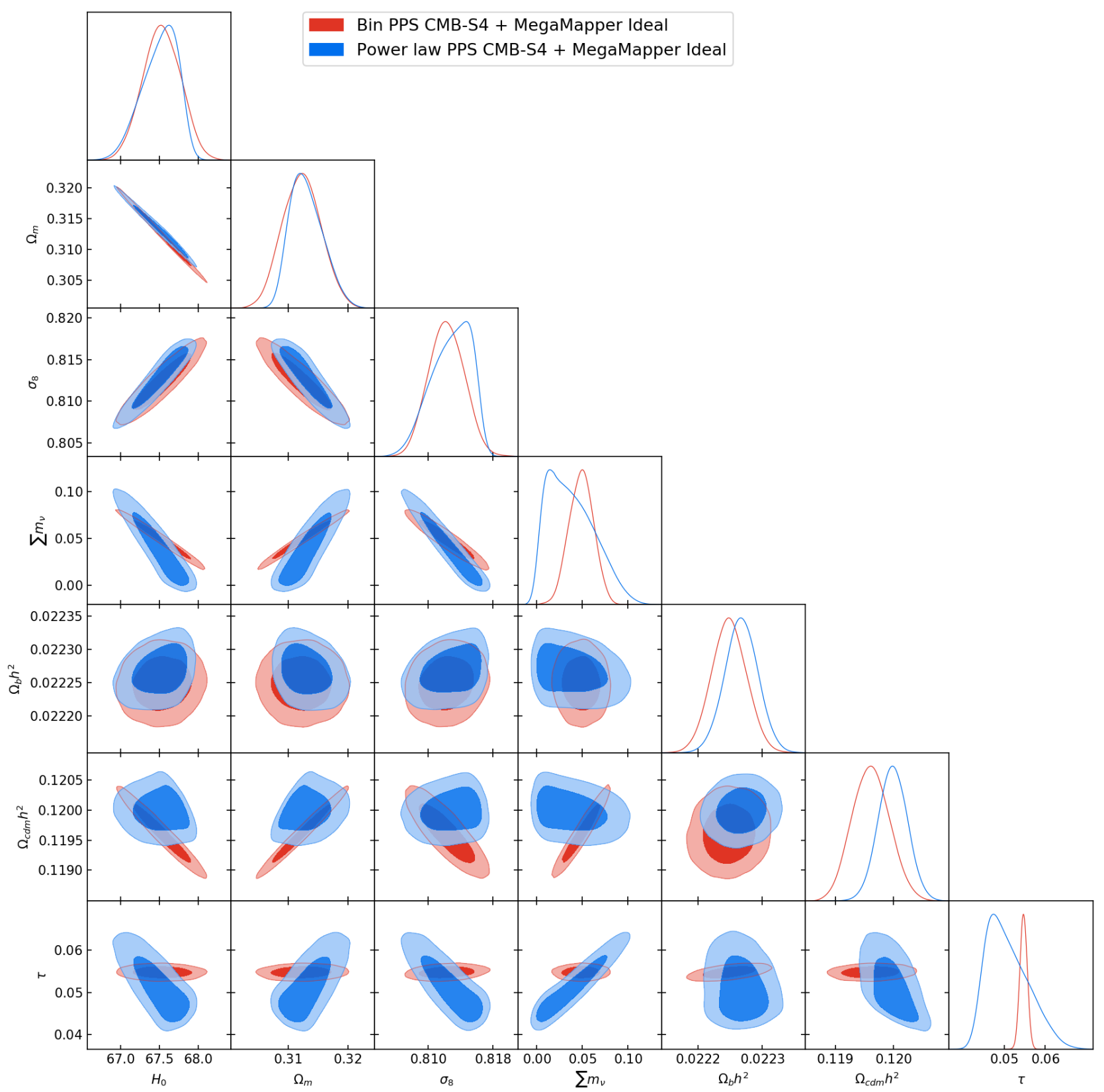

FIG. 11. Corner plot of cosmology parameters for CMB-S4 plus MegaMapper Ideal data, comparing fits allowing PPS freedom vs fixing to power law behavior.

[26] B. L'Huillier, A. Shafieloo, D. K. Hazra, G. Smoot, and A. Starobinsky, Probing Features in the Primordial Perturbation Spectrum with Large-Scale Structure Data, Mon. Not. Roy. Astron. Soc. 477 (2018), 2503, arXiv:1710.10987.

[27] G. Obied, C. Dvorkin, C. Heinrich, W. Hu, and V. Miranda, Inflationary Features and Shifts in Cosmological Parameters from Planck 2015 Data, Phys. Rev. D 96 (2017), 083526, arXiv:1706.09412.

[28] Planck Collaboration, Planck 2018 results. VI. Cosmological parameters, arXiv:1807.06209.

[29] D. Blas, J. Lesgourgues, T. Tram, CLASS II: Approximation schemes, JCAP 1107 (2011) 034, arXiv:1104.2933.

[30] A. Achúcarro, V. Atal, P. Ortiz, and J. Torrado, Localized correlated features in the CMB power spectrum and primordial bispectrum from a transient reduction in the speed of sound, Phys. Rev. D 89 (2014), 103006, arXiv:1311.2552.

[31] F.-Y. Cyr-Racine, R. de Putter, A. Raccanelli and K. Sigurdson, Constraints on Large-Scale Dark Acoustic Oscillations from Cosmology, Phys. Rev. D 89 (2014), 063517, arXiv:1310.3278.

[32] CMB-S4 collaboration, CMB-S4 Science Book, First Edition, arXiv:1610.02743.

[33] K. Abazajian et al., CMB-S4 Science Case, Reference Design, and Project Plan, arXiv:1907.04473. 


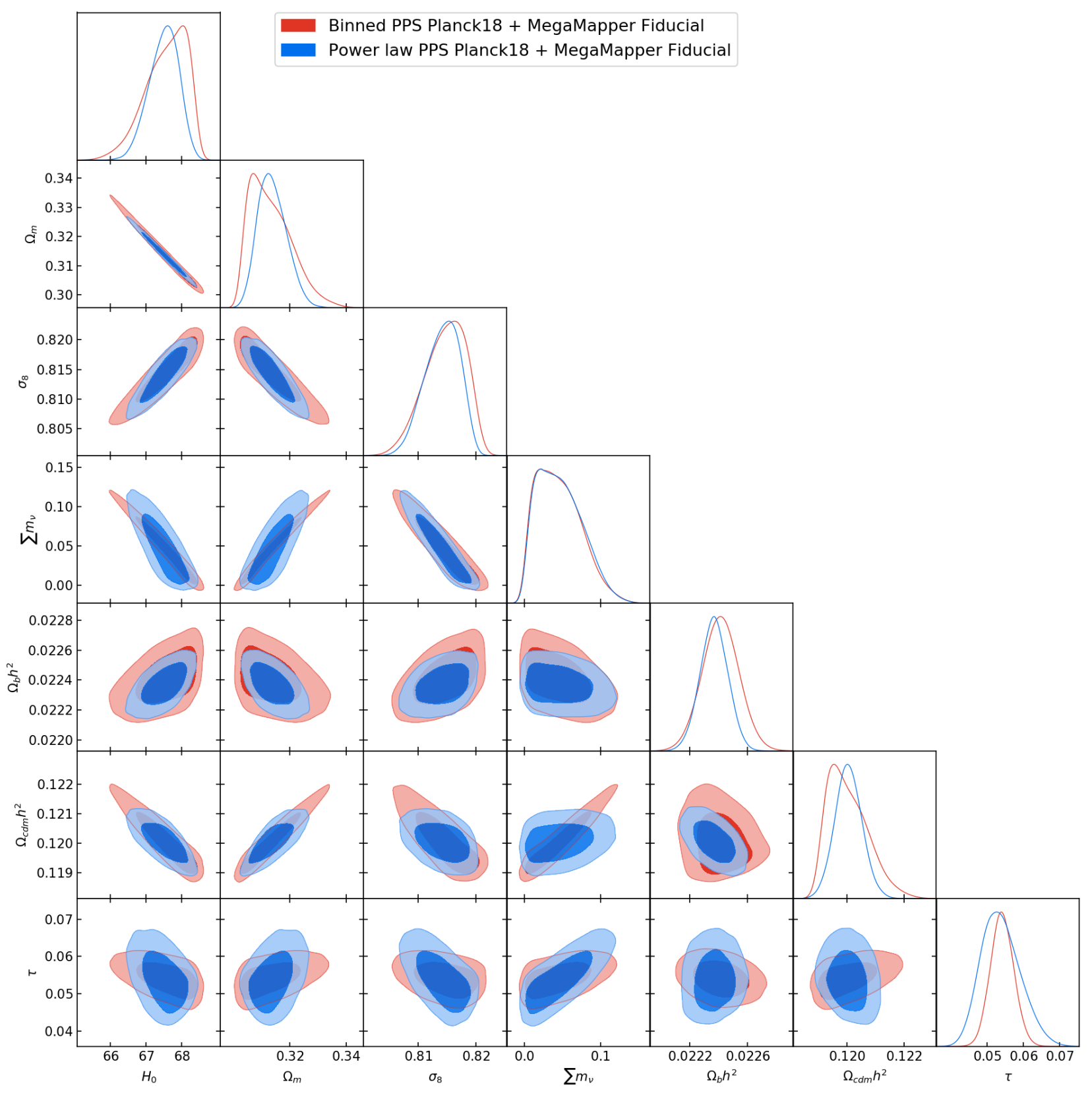

FIG. 12. As Fig. 11, but for Planck18 plus MegaMapper Fiducial.

[34] D.J. Schlegel et al., Astro2020 APC white paper: the MegaMapper: a $z>2$ spectroscopic instrument for the study of inflation and dark energy, arXiv:1907.11171.

[35] S. Ferraro et al., Inflation and Dark Energy from spectroscopy at $z>2$, arXiv:1903.09208.

[36] B. Audren, J. Lesgourgues, K. Benabed, and S. Prunet, Conservative constraints on early cosmology: An illustration of the Monte Python cosmological parameter inference code, JCAP 1302 (2013) 001, arXiv:1210.7183.

[37] T. Brinckmann and J. Lesgourgues, MontePython 3: Boosted MCMC sampler and other features, Phys. Rev. D 97 (2018), 063506, arXiv:1804.07261.

[38] T. Brinckmann, D. C. Hooper, M. Archidiacono, J. Lesgourgues, and T. Sprenger, The promising future of a robust cosmological neutrino mass measurement, JCAP 1901 (2019) 059, arXiv:1808.05955.

[39] A. Lewis, GetDist: a Python package for analysing Monte Carlo samples, arXiv:1910.13970, https://getdist.readthedocs.io.

[40] T. Matsumura et al., Mission design of LiteBIRD, Journal of Low Temperature Physics, 176 (2014) 733, arXiv:1311.2847.

[41] H. Sugai et al., Updated design of the CMB polarization experiment satellite LiteBIRD, Journal of Low Temperature Physics (2020), in press, arXiv:2001.01724. 